\title{
Choledocholithiasis Without Cholelithiasis: Should the Gallbladder Stay or Should It Go?
}

\author{
Pritesh Mutha $^{1} \cdot$ Tilak Shah $^{1} \cdot$ Douglas Heuman $^{1} \cdot$ Alvin Zfass $^{1} \cdot$ Mitchell L. Schubert $^{1}$
}

Published online: 6 January 2016

(C) Springer Science+Business Media New York (Outside the USA) 2016

Cholelithiasis (gallstones) is common, with an estimated prevalence of $\sim 12 \%$ of adult population in the USA. The prevalence, increasing with age, approaches $33 \%$ in elderly women [1]. Approximately $15 \%$ of patients with cholelithiasis have concurrent choledocholithiasis (bile duct stones), and conversely, $95 \%$ of patients with choledocholithiasis have cholelithiasis [2, 3]. Although the natural history of asymptomatic common bile duct (CBD) stones is not well defined, they are thought to be less benign than asymptomatic gallbladder gallstones. Although CBD stones may pass uneventfully through the ampulla of Vater into the duodenum, their passage can induce acute pancreatitis and stone impaction at the distal bile duct, causing biliary colic and cholangitis. Because adverse events may be serious and life-threatening, it is generally recommended that CBD stones be removed.

Over the last several decades, endoscopic retrograde cholangiopancreatography (ERCP) with sphincterotomy has emerged as the procedure of choice to remove CBD stones. Sphincterotomy may be performed with or without balloon dilation. After removal of CBD stones, cholecystectomy is typically performed, unless the patient has significant comorbid conditions. The rationale for this approach is that (1) cholesterol CBD stones, which represent $>75 \%$ of bile duct stones in the USA and Europe, originate in the gallbladder and (2) gallbladder function is impaired in patients with cholesterol gallstones and persists

Pritesh Mutha

Pritesh.Mutha@va.gov

1 Division of Gastroenterology (Code: 111N), Department of Medicine, Hunter Holmes McGuire Veterans Affairs Medical Center, Virginia Commonwealth University Health System, 1201 Broad Rock Blvd, Richmond, VA 23249, USA after the removal of stones [4]. Thus, unless expected lifespan is short, cholelithiasis and subsequent choledocholithiasis are predicted to recur. Indeed, a meta-analysis of five randomized controlled trials comparing cholecystectomy after endoscopic sphincterotomy to a wait-and-see approach reported a significantly higher risk of biliary complications and death in the latter group in the five studies [5].

Unlike cholesterol stones that originate in the gallbladder, brown pigment stones, common in Asia (e.g., Korea, Japan, China, Vietnam, Taiwan, and Southeast Asia), may arise de novo within the bile duct, including the intrahepatic ducts. These stones, composed of calcium bilirubinate, may account for up to $30 \%$ of ductal stones in these regions and are associated with recurrent cholangitis, biliary cirrhosis, and cholangiocarcinoma [6]. The precise etiology is unknown, but infection of the bile ducts by parasites such as liver flukes (e.g., Clonorchis sinensis) and parasitic worms is thought to be contributory. Bile stasis, induced by the parasites and their ova, is postulated to lead to bacterial colonization. Bacterial enzymes, in turn, hydrolyze bilirubin conjugates and phospholipids in bile, releasing free bilirubin and fatty acids that form insoluble precipitates with biliary calcium. The resulting concretions are termed brown pigment stones [7]. The necessity of cholecystectomy after ERCP extraction of isolated brown bile duct stones is less clear. In fact, none of the individual studies in the meta-analysis described earlier specifically reports the outcomes of patients with isolated bile duct stones in the absence of cholelithiasis [5].

In the current issue of Digestive Diseases and Sciences, Kim et al. [8] retrospectively describe the natural history of 453 South Korean patients with bile duct stones who underwent ERCP with sphincterotomy and/or balloon dilation for stone removal between 1998 and 2000, but did 
not undergo cholecystectomy. Concurrent cholelithiasis was present in $256(57 \%)$ patients and absent in 197 (43\%). As is typical for this population, the majority of stones in both groups were brown pigment; only $5-11 \%$ of patients had cholesterol stones. Patients with cholelithiasis who did not undergo cholecystectomy had either declined surgery or had comorbidities that portended a prohibitive operative risk. The investigators followed these patients for a mean duration of 785 days. Gallbladder complications (biliary colic, acute cholecystitis, and gallbladder cancer) occurred in $29(11.3 \%)$ patients in the calculous gallbladder group compared to $5(2.5 \%)$ patients in the acalculous gallbladder group $(p=0.001)$. The incidence of recurrent bile duct stones, with or without cholangitis, did not differ significantly in patients with or without gallbladder stones (11.3 and $10.2 \%$, respectively). No gallbladder- or bile duct stone-related mortality occurred in any of the patients. The authors conclude that prophylactic cholecystectomy may not be necessary after clearing pigment stones from the bile duct if the patient does not have concurrent cholelithiasis.

Should physicians recommend cholecystectomy after endoscopic removal of isolated CBD stones or adopt a wait-and-see approach? The results of the study are certainly consistent with that of several other non-randomized retrospective publications from Asia [9]. To place in perspective the $2.5 \%$ risk of gallbladder-related complications reported in the present study, the overall perioperative morbidity for elective cholecystectomy is $\sim 10 \%$ [3]. Despite the retrospective nature of these studies, it does appear that a wait-and-see approach is reasonable in patients with isolated CBD pigment stones.

Nevertheless, Can we extrapolate these conclusions to a Western population? Cholesterol stones account for the vast majority of stones in Western countries (either pure cholesterol or mixed stones that contain at least $50 \%$ cholesterol by weight). In this population, stones originate almost exclusively in the gallbladder; choledocholithiasis results when gallbladder stones pass through the cystic duct. Consequently, isolated bile duct cholesterol stones may indeed be a misnomer as patients may have unrecognized gallbladder sludge or microlithiasis that by itself can induce cholecystitis, cholangitis, and pancreatitis as well as develop into larger stones that may pass into the CBD. Although ursodeoxycholic acid (UDCA) treatment can reduce the amount of biliary sludge and its complications [10], once it is discontinued, sludge and stones return in $>50 \%$ of patients over 5-12 years [11].

Pending a well-designed prospective trial, we recommend, at least in Western populations, elective cholecystectomy in patients with an acalculous gallbladder following removal of CBD stones. In high-risk patients and in older adults, individualized management (i.e., a waitand-see approach) is reasonable. In those with sludge or small stones, UDCA therapy may be considered.

\section{References}

1. Everhart JE, Khare M, Hill M, Maurer KR. Prevalence and ethnic differences in gallbladder disease in the United States. Gastroenterology. 1999;117:632-639.

2. Ebner S, Rechner J, Beller S, Erhart K, Riegler F, Szinicz G. Laparoscopic management of common bile duct stones. Surg Endosc. 2004;18:762-765. doi:10.1007/s00464-003-9029-5.

3. Velanovich V, Morton J, McDonald M, Orlando R, Maupin G, Traverso L. Analysis of the SAGES outcomes initiative cholecystectomy registry. Surg Endosc. 2006;20:43-50. doi:10.1007/ s00464-005-0378-0.

4. Stolk MFJ, Van Erpecum KJ, Renooij W, Portincasa P, Heijning VD, vanBerge-Henegouwen G. Gallbladder emptying in vivo, bile composition, and nucleation of cholesterol crystals in patients with cholesterol gallstones. Gastroenterology. 1995;108:1882-1888. doi:10.1016/0016-5085(95)90153-1.

5. Mcalister VC, Davenport E, Renouf E. Cholecystectomy deferral in patients with endoscopic sphincterotomy. The Cochrane Database of Systematic Reviews 2007; CD006233.

6. Kim HG, Han J, Kim M-H, et al. Prevalence of clonorchiasis in patients with gastrointestinal disease: a Korean nationwide multicenter survey. World J Gastroenterol. 2009;15:86-94.

7. Maki T. Pathogenesis of calcium bilirubinate gallstone: role of E. coli, beta- glucuronidase and coagulation by inorganic ions, polyelectrolytes and agitation. Ann Surg. 1966;164:90.

8. Kim M, Yeo S, Jung M, Cho C. The impact of gallbladder status on biliary complications after the endoscopic removal of choledocholithiasis. Dig Dis Sci. (Epub ahead of print). doi:10.1007/ s10620-015-3915-2.

9. Tsai T, Lai K, Lin C, et al. The relationship between gallbladder status and recurrent biliary complications in patients with choledocholithiasis following endoscopic treatment. J Chin Med Assoc. 2012;75:560-566. doi:10.1016/j.jcma.2012.08.003.

10. Portincasa P, Ciaula AD, Bonfrate L, Wang DQ. Therapy of gallstone disease: what it was, what it is, what it will be. World $J$ Gastrointest Pharmacol Ther. 2012;3:7-20. doi:10.4292/wjgpt. v3.i2.7.

11. Villanova N, Bazzoli F, Taroni F, et al. Gallstone recurrence after successful oral bile acid treatment: a 12- year follow- up study and evaluation of long- term postdissolution treatment. Gastroenterology. 1989;97:726-731. 